Publ. RIMS, Kyoto Univ.

17 (1981), 311-330

\title{
A Generalization of Vanishing Theorems for Weakly 1-Complete Manifolds
}

\author{
By \\ Kensho TAKEGOSH ${ }^{*}$
}

\section{$\S 1$ Introduction}

Let $X$ be a connected complex manifold of complex dimension $n . \quad X$ is called weakly l-complete if there exists an exhaustion function $\Phi$ on $\mathrm{X}$ which is $C^{\infty}$ and plurisubharmonic. In [9] S. Nakano established the following.

Theorem 1. Let $B$ be a positive line bundle on a weakly 1complete manifold $X$, then

$$
H^{p}\left(X, \Omega^{q}(B)\right)=0 \quad \text { for } \quad p+q>n .
$$

Recently, O. Abdelkader obtained

Theorem 2 (cf. [1]). Let $B$ be a semi-positive line bundle over a weakly 1-complete Kähler manifold $\mathrm{X}$ and assume that the curvature form of $B$ has at least $n-k+1$ positive eigenvalues, then

$$
H^{p}\left(X_{c}, \Omega^{q}(B)\right)=0 \text { for any real number } c \text { with } p+q \geqq n+k \text {, }
$$

where $X_{c}=\left\{x \in X_{i} \Phi(x)<c\right\}$.

In these theorems, the positivity of eigenvalues of the curvaturc form of $B$ is assumed on the whole space $X$. In this paper, we shall prove that these vanishing theorems still hold, if the positivity of eigenvalues of the curvature admits a compact exceptional subset $K \subsetneq X$. We shall prove the following.

Communicated by S. Nakano, July 1, 1980.

* Research Institute for Mathematical Sciences, Kyoto University, Kyoto 606, Japan. 
Main Theorem. Let $B$ be a semi-positive line bundle over a connected weakly 1-complete Kähler manifold $X$ with a metric along. the fibres such that its curvature form has at least $n-q+1$ positive eigenvalues on $X \backslash K$, where $K$ is a proper compact subset of $X$. Then

$$
H^{p}\left(X, \mathcal{O}\left(B \otimes K_{X}\right)\right)=0 \text { for any } p \geqq q \text {, }
$$

where $K_{X}$ is the canonical line bundle of $X$.

In particular, when $q=1$, we obtain

Corollary. Let $X$ be a connected weakly 1-complete Kähler manifold and let $B$ be a semi-positive line bundle on $X$ which is positive on $X \backslash K$ for some proper compact subset $K$ of $X$. Then

$$
H^{p}\left(X, \Theta\left(B \otimes K_{X}\right)\right)=0 \quad \text { for any } p \geqq 1 \text {. }
$$

Since a positive line bundle over a complex manifold induces a Kähler metric on it, this is not only a direct generalization of Theorem 1 for $q=n$ but also a generalization of the vanishing theorems for the semi-positive line bundle on 1-convex Kähler manifolds and compact Kähler manifolds by Grauert and Riemenschneider (cf. [4], [11]).

This work is inspired by Ohsawa's article [10] and the author would like to express his hearty thanks to Dr. A. Fujiki and Professor S. Nakano for their kind advices and encouragement during the preparation of this paper.

\section{$\S$ 2. Notations and Definitions}

We denote by $X$ a connected paracompact complex manifold of dimension $n$. Let $\pi: F \rightarrow X$ be a holomorphic line bundle over $X$. Let $\mathcal{U}=\left\{U_{i}\right\}_{i \in I}$ be a covering of $X$ by coordinate neighborhoods such that on each $U_{i}, F \mid U_{i}$ is isomorphic to the trivial line bundle. We denote local coordinates on $U_{i}$ by $\left(z_{i}^{1}, \cdots, z_{i}^{n}\right)$. If $\Phi_{i}: U_{i} \times \boldsymbol{C} \rightarrow F \mid U_{i}(i \in I)$ are these trivializations of $F$, we denote by $f_{i j}: U_{i} \cap U_{j} \rightarrow \mathbb{C}^{*}$ the system of transition functions defined by the conditions:

$$
\Phi_{j}^{-1} \circ \Phi_{i}\left(z_{i}, \xi_{i}\right)=\left(z_{i}, f_{i j}\left(z_{j}\right) \xi_{j}\right)
$$


where $\xi_{i}$ denotes the fibre coordinates over $U_{i}$.

An $F$-valued differential form $\varphi$ on $X$ is a system $\left\{\varphi_{i}\right\}_{i \in I}$ of differential forms defined on $U_{i}$, satisfying $\varphi_{i}=f_{i j} \varphi_{j}$ in $U_{i} \cap U_{j}$. We denote by $C^{p, q}(X, F)$ the space of $F$-valued differential forms on $X$, of class $C^{\infty}$ and of type $(p, q)$, and by $C_{0}^{p, q}(X, F)$ the space of the forms in $C^{p, q}(X, F)$ with compact supports.

Let $d s^{2}=\sum_{\alpha, \beta=1}^{n} g_{i, \alpha \bar{\beta}} d z_{i}^{a} \cdot d z_{i}^{\bar{\beta}}$ be a hermitian metric on $X$ and let $\left\{a_{i}\right\}$ be a hermitian metric along the fibres of $F$, that is, a system of positive valued function $a_{i}$ in $U_{i}$ satisfying $\left|f_{i j}\right|=a_{i} \cdot a_{j}^{-1}$ in $U_{i} \cap U_{j}$.

Remark. In this paper, we use the notation of a system of metrics along the fibres in the sense of Kodaira [7], page 1268, (1).

For $\varphi, \psi \in \mathrm{C}^{p, q}(X, F)$, we set

$$
\langle\varphi, \psi\rangle=a_{i}^{-1} \sum_{A_{p}, B_{q}} \varphi_{i A_{p}, \vec{B}_{q}} \cdot \bar{\psi}_{i}^{\bar{A}, B_{q}},
$$

where $\varphi_{i}=\sum_{A_{p}, B_{q}} \varphi_{i A_{p}, \bar{B}_{q}} d z_{i}^{A_{p}} \wedge d z_{i}^{\bar{B} q}$ and $A_{p}=\left(\alpha_{1}, \cdots, \alpha_{p}\right)$ and $B_{q}=\left(\beta_{1}, \cdots, \beta_{q}\right)$ run through the sets of multi-indices with $1 \leqq \alpha_{1}<\cdots<\alpha_{p} \leqq n$ and $1 \leqq \beta_{1}<$ $\cdots<\beta_{q} \leqq n$ respectively. Then

$$
a_{i}^{-1} \varphi_{i} \wedge * \overline{\phi_{i}}=\langle\varphi, \phi\rangle d V
$$

where $*$ is the star operator and $d V$ is the volume element with respect to the metric $d s^{2}$.

If either $\varphi$ or $\psi \in C_{0}^{p, q}(X, F)$, we define

$$
(\varphi, \psi)_{r}=\int_{X}\langle\varphi, \psi\rangle e^{-\psi} d V
$$

for any real-valued $C^{\infty}$-function $\Psi$.

In particular we set

$$
(\varphi, \psi)=(\varphi, \psi)_{0}
$$

and

$$
\begin{aligned}
\|\varphi\|_{\Psi}^{2} & =(\varphi, \varphi)_{r} \\
\|\varphi\|^{2} & =(\varphi, \varphi) .
\end{aligned}
$$

We have the operator $\bar{\partial}: C^{p, q}(X, F) \rightarrow C^{p, q+1}(X, F)$ defined by $(\bar{\partial} \varphi)_{i}$ 
$=\bar{\partial} \varphi_{i}$. With respect to (2.1) and (2.2), the formal adjoint operator of $\bar{\partial}$ are defined, we denote them by $\vartheta_{\Psi}$ and $\vartheta$ respectively. We denote by $L^{p, q}(X, F, \Psi)$ (resp. $\left.L^{p, q}(X, F)\right)$ the space of the measurable $F$-valued forms $\varphi$ of type $(p, q)$, square integrable in the sense that $\|\varphi\|_{\Psi^{2}}^{2}<\infty$ (resp. $\left.\|\varphi\|^{2}<\infty\right)$. Then, they are Hilbert spaces with respect to the inner product $(\varphi, \psi)_{1}$ (resp. $\left.(\varphi, \psi)\right)$. We denote again by $\bar{\partial}$ the operator from $L^{p, q}(X, F, \Psi)$ to $L^{p, q+1}(X, F, \Psi)$ extending the original $\bar{\partial}$; thus a form $\varphi \in L^{p, q}(X, F, \Psi)$ is in the domain of $\bar{\partial}$ if and only if $\bar{\partial} \varphi$, defined in the sense of distribution, belongs to $L^{p, q-1}(X, F, \Psi)$. Then $\bar{\partial}$ is a closed, densely defined operator, so the adjoint operator $\bar{\partial}_{T}^{*}$ (resp. $\bar{\partial}^{*}$ ) can be defined. We denote the domain, range and nullity of $\bar{\partial}$ in $L^{p, q}(X, F, \Psi)$

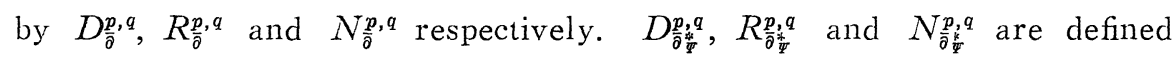
similarly.

Definition 2. 1. $X$ is called weakly 1 -complete if there exists a $C^{\infty}$-plurisubharmonic function $\Phi$ on $X$ such that for any real number $c$, $X_{c}=\{x \in X \mid \Phi(x)<c\}$ is relatively compact in $X$.

Remark 2.1. Let $\lambda(t):(-\infty, \infty) \rightarrow(-\infty, \infty)$ be a $C^{\infty}$-increasing convex function such that $\lambda(t)=0$ for $t \leqq 0$, then the composition $\lambda(\Phi)$ is again $C^{\infty}$-plurisubharmonic and exhausts $X$. So we may assume that $\Phi$ is non-negative on $X$. Then, for any $c \in(0, \infty), X_{c}=\{x \in X \mid \Phi(x)<c\}$ is weakly 1 -complete with respect to the exhaustion function $\frac{1}{c-\Phi}$.

Remark 2.2. Any connected compact complex manifold is weakly 1-complete, any real constant function being taken as the exhaustion function.

Definition 2. 2. A holomorphic line bundle $\pi: F \rightarrow X$ is said to be positive (resp. semi-positive) on a subset $Y \subset X$, if there exist a coordinate cover $q=\left\{U_{i}\right\}_{i \in I}$ of $X$ such that $\pi^{-1}\left(U_{i}\right)$ are trivial and a metric $\left\{a_{i}\right\}$ along the fibres of $F$ such that

$$
\left(\frac{\partial^{2} \log a_{i}}{\partial z_{i}^{\alpha} \partial \bar{z}_{i}^{\beta}}\right)>0 \quad(\text { resp. } \geqq 0) \quad \text { on } U_{i} \cap Y \text { for every } i \in I
$$


Definition 2. 3. A holomorphic line bundle $\pi: F \rightarrow X$ is said to be $q$-semi-positive $(1 \leqq q \leqq n)$ on a subset $Y \subset X$, if $F$ is semi-positive on $Y$ and the hermitian matrix (2.4) has at least $n-q+1$ positive eigenvalues at cach point of $Y$.

\section{§3. A Formulation of $\mathbb{L}^{2}$-Estimates and Existence Theorems for the $\bar{\partial}$ Operator}

Let $\mathrm{X}$ be a paracompact complex manifold of dimension $n$ which is nnt necessarily connected.

Theorem 3. 1. Let $F$ be a holomorphic line lundle over $\mathrm{X}$. If there exist in the degree $(p, q)$

(3.1) a complete hermitian metric $d s^{2}$ on $\mathrm{X}$,

(3.2) a hermitian metric $\left\{a_{i}\right\}$ along the fibres of $F$,

(3. 3) a constanl $C_{1}>0$

and

(3. 4) a compact subset $K$ of $X$ rohich docs not contain any connected component of $X$, such that

$$
\|\varphi\|_{X \backslash K}^{2} \leqq C_{1}\left\{\|\bar{\partial} \varphi\|^{2}+\left\|\bar{\partial}^{*} \varphi\right\|^{2}\right\} \text { for } a n y^{\prime} \varphi \in D_{\bar{\partial}}^{p \cdot q} \cap D_{\bar{\partial}^{\prime}{ }^{p}}^{p} .
$$

Then, there exists a constant $C_{2}>0$ such that

$$
\|\varphi\|^{2} \leqq C_{2}\left\{\|\bar{\partial} \varphi\|^{2}+\left\|\bar{\partial}^{*} \varphi\right\|^{2}\right\} \quad \text { for any } \varphi \in D_{\bar{\partial}}^{p, q} \cap D_{\bar{\partial}, q}^{p, q} .
$$

Proof. Take any sequence $\left\{\varphi_{m}\right\}$ such that $\varphi_{n} \in D_{\bar{\partial}}^{p, q} \cap D_{\bar{\partial}^{q}}^{p},\left\|\varphi_{m}\right\|^{2} \leqq 1$, $\lim _{m \rightarrow+\infty}\left\|\bar{\partial} \varphi_{m}\right\|^{2}=0$ and $\lim _{m \rightarrow+\infty}\left\|\bar{\partial}^{*} \varphi_{m}\right\|^{2}=0$. Then we assert that there exists a subsequence $\left\{\varphi_{m_{h}}\right\}$ of $\left\{\varphi_{m}\right\}$ which converges strongly on $X$. Since $d s^{2}$ is complete, $C_{n}^{p, q}(X, F)$ is dense in $D_{\bar{\partial}}^{p, q} \cap D_{\bar{\partial}}^{p, q}$ with respect to the norm

$$
(\bar{\partial} \varphi, \bar{\partial} \varphi)+\left(\bar{\partial}^{*} \varphi, \bar{\partial}^{*} \varphi\right)+(\varphi, \varphi)
$$

([12], Theorem 1.1). Hence we may assume $\varphi_{m} \in C_{0}^{p, q}(X, F)$. Therefore we obtain that

$$
\begin{array}{r}
\left(\bar{\partial} \varphi_{m}, \bar{\partial} \varphi_{m}\right)+\left(\bar{\partial} * \varphi_{m}, \bar{\partial} * \varphi_{m}\right) \mid\left(\varphi_{m}, \varphi_{m}\right) \\
=\left((\bar{\partial} \vartheta+\vartheta \bar{\partial}) \varphi_{m}, \varphi_{m}\right)+\left(\varphi_{m}, \varphi_{m}\right)
\end{array}
$$


is bounded by the assumption. Since $\bar{\partial} \vartheta+\vartheta \bar{\partial}$ is an elliptic differential operator of order 2 , this means that $\left(\varphi_{m}\right)_{i}$ and their first derivatives with respect to the coordinate of $U_{i}$ are bounded in the sense of the integral $\|,\|_{K^{\prime}}^{2}$, where $K^{\prime}$ is a compact subset of $X$ with $K \subset \operatorname{Int} K^{\prime}$ (see for example [3], (2.2.1) Theorem). Combining this with Rellich's lemma (see for example [3], Appendix), it follows that $\left\{\varphi_{m}\right\}$ has a subsequence $\left\{\varphi_{m_{k}}\right\}$ which is strongly convergent on compact subsets. By (3.5), we conclude that $\left\{\varphi_{m_{k}}\right\}$ converges strongly on $X$. Therefore, by Hörmander [5] Theorem 1.1.2 and Theorem 1.1.3, there exists a positive constant $C_{2}$ such that

$$
\|\varphi\|^{2} \leqq C_{2}\left\{\|\bar{\partial} \varphi\|^{2}+\left\|\bar{\partial}^{*} \varphi\right\|^{2}\right\}
$$

for any $\varphi \in D_{\overline{\bar{\partial}}}^{p, q} \cap D_{\overline{\bar{\sigma}}^{*}}^{p}$, with $\varphi \perp N^{p, q}=N_{\overline{\bar{\partial}}}^{p, q} \cap N_{\bar{\partial}^{*}}^{p}$.

By the same theorems we obtain the following strong orthogonal decomposition:

$$
L^{p, q}(X, F)=R_{\overline{\bar{\partial}}}^{p, q} \oplus N^{p, q} \oplus R_{\bar{\partial}^{*}}^{p, q} .
$$

Each element $\varphi$ in $N^{p, q}$ is a solution of the Laplace-Beltrami operator $\square=\bar{\partial} \vartheta+\vartheta \bar{\partial}$ with respect to (3.1) and (3.2). Now we refer to the unique continuation theorem for harmonic forms with values in a hermitian vector bundle.

Theorem 3.2 (Aronszajn [2], Riemenschneider [11]). Let E be a hermitian vector bundle over a connected complex hermitian manifold $X$. Then a harmonic form $\varphi \in \mathcal{H}^{p, q}(E)$ vanishes identically on $X$ if it vanishes on a non-empty open subset $U$ of $X$.

Any form $\varphi$ in $N^{p, q}$ vanishes on the open subset $X \backslash K$ by (3.5). Since each connected component of $X$ is not contained in $K$ by the assumption, from Theorem 3.2, $\varphi$ vanishes identically on each connected component. Hence $\varphi$ vanishes identically on $X$. Therefore $N^{p, q}$ is the null space. Combining this with (3.7), our theorem follows. q.e.d.

From the above theorem, we obtain (cf. [5], Theorem 1.1.4)

Corollary 3.1. Let $X, F$ and others be as above. Let $\varphi \in L^{p, q}$ 
$(X, F)$ satisfy the equation $\bar{\partial} \varphi=0$, then there exists $a \psi \in L^{p, q-1}(X, F)$ such that $\bar{\partial} \psi=\varphi$. Moreover, if $\varphi \in C^{p, q}(X, F)$, then $\psi$ can be taken from $C^{p, q-1}(X, F)$ (cf. [6], p. 115, Theorem 5.2.5).

\section{\$4. The Basic Estimate}

Let $X$ be a connected paracompact complex manifold of dimension $n$ and let $\pi: B \rightarrow X$ be a holomorphic line bundle over $X$. Let $U=\left\{U_{i}\right\}_{i \in I}$ be a coordinate cover of $X$ such that $\pi^{-1}\left(U_{i}\right)$ are trivial and let $\left\{a_{i}\right\}$ be a hermitian metric along the fibres of $B$ with respect to $U$. We set

$$
\Gamma_{i, \alpha \bar{\beta}}=\frac{\partial^{2} \log a_{i}}{\partial z_{i}^{\alpha} \partial z_{i}^{\bar{\beta}}} .
$$

We assume that $X$ is provided with a Kähler metric

$$
d s^{2}=\sum_{\alpha, \beta=1}^{n} g_{i, \alpha \bar{\beta}} d z_{i}^{\alpha} \cdot d z_{i}^{\bar{\beta}} .
$$

The canonical line bundle $K_{X}$ of $X$ is defined by a system of transition functions $\left\{K_{X, i j}\right\}$ on $U_{i} \cap U_{j}$, where $K_{X, i j}=\frac{\partial\left(z_{j}^{1}, \cdots, z_{j}^{n}\right)}{\partial\left(z_{i}^{1}, \cdots, z_{i}^{n}\right)}$. Then we see that

$$
\left|K_{X, i j}\right|^{2}=g_{i} \cdot g_{j}^{-1} \quad \text { on } U_{i} \cap U_{j}
$$

where

$$
g_{i}=\operatorname{det}\left(g_{i, \alpha \bar{\beta}}\right)
$$

Hence $\left\{g_{i}\right\}$ determines a metric along the fibres of $K_{X}$. Then $\left\{A_{i}\right\}$ defined by

$$
A_{i}=a_{i} \cdot g_{i}
$$

determines a metric of $B \otimes K_{X}$.

With the notations (2.1), (2.2) and (2.3), the following inequality has been shown by K. Kodaira (cf. [7], pp. 1269-1270).

$$
\int_{X} \frac{1}{A_{i}} \sum_{B_{p-1}} \sum_{\beta, \gamma=1}^{n} \Gamma_{i, \beta \bar{\gamma}} \varphi_{i \bar{B}_{p-1}} \cdot \overline{\varphi_{i}^{\tau \bar{B}_{p-1}}} d V \leqq\|\bar{\partial} \varphi\|^{2}+\left\|\bar{\partial}^{*} \varphi\right\|^{2}
$$

for any $\varphi \in C_{0}^{0, p}\left(X, B \otimes K_{X}\right)$ with $p \geqq 1$.

From now on, we let $X$ be a connected Kähler manifold, weakly 1-complete with respect to an exhaustion function $\Phi$ and let $\pi: B \rightarrow X$ be 
a holomorphic line bundle which is semi-positive on $X$ and $q$-semi-positive on $X \backslash K$ for some proper compact subset $K$ of $X$. We fix a constant $c>c_{*}=\sup _{x \in K} \Phi(x)$. Then $X_{c}=\{x \in X \mid \Phi(x)<c\}$ is weakly 1-complete with respect to the exhaustion function $\frac{1}{c-\Phi}$.

We take a Kähler metric

$$
d s_{0}^{2}=\sum_{\alpha, \beta=1}^{n} g_{i, \alpha \bar{\beta}, 0} d z_{i}^{\alpha} \cdot d z_{i}^{\bar{\beta}}
$$

on $X$. We set

$$
G_{i, 0}=\left(g_{i, \alpha \bar{\beta}, 0}\right) \text {. }
$$

Let $\left\{a_{i, 0}\right\}$ be a fibre metric of $B$ which corresponds to the assumption and we set

$$
\Gamma_{i, 0}=\left(\Gamma_{i, \alpha \bar{\beta}, 0}\right) \quad \text { where } \quad \Gamma_{i, \alpha \bar{\beta}, 0}=\frac{\partial^{2} \log a_{i, 0}}{\partial z_{i}^{\alpha} \partial z_{i}^{\bar{\beta}}}
$$

We can assume that $\inf _{x \in X} \Phi(x)=0$. Then we take a $C^{\infty}$ increasing convex function $\lambda(t)$ such that

$$
\begin{aligned}
& \text { i) } \lambda(t):(-\infty, \infty) \rightarrow(-\infty, \infty), \\
& \text { ii) } \lambda(t)=\left\{\begin{aligned}
0 & \text { if } t \leqq \frac{1}{c} \\
>0 & \text { if } t>\frac{1}{c},
\end{aligned} \text { iii) } \quad \int_{0}^{+\infty} \sqrt{\lambda^{\prime \prime}(t)} d t=+\infty .\right.
\end{aligned}
$$

We replace the metric along the fibers of $B$ by

$$
a_{i}=a_{i, 0} \cdot \exp (\Psi) \quad \text { where } \Psi=\lambda\left(\begin{array}{c}
1 \\
c-\Phi
\end{array}\right)
$$

We set

$$
\Gamma_{i}=\left(\Gamma_{i, \alpha \bar{\beta}}\right) \quad \text { where } \quad \Gamma_{i, \alpha \bar{\beta}}=\frac{\partial^{2} \log a_{i}}{\partial z_{i}^{\alpha} \partial z_{i}^{\bar{\beta}}}
$$

Then we have

$$
\Gamma_{i} \geqq \Gamma_{i, 0}
$$


We define a Kähler metric $d s^{2}$ by

$$
d s^{2}=\sum_{\alpha, \beta=1}^{n}\left(g_{i, \alpha \bar{\beta}, 0}+\Gamma_{i, \alpha \bar{\beta}}\right) d z_{i}^{\alpha} \cdot d z_{i}^{\bar{\beta}} .
$$

Remark. By the choice of $\lambda$ as in (4.9) iii), $d s^{2}$ is a complete Kähler metric on $X_{c}$ (cf. [8], Proposition 1).

We set

$$
G_{i}=\left(g_{i, \alpha \bar{\beta}}\right) \quad \text { where } \quad g_{i, \alpha \bar{\beta}}=g_{i, a \bar{\beta}, 0}+\Gamma_{i, \alpha \bar{\beta}} .
$$

We replace the metric along the fibres of $B \otimes K_{Y}$ by

$$
A_{i}=a_{i} \cdot g_{i} \text { where } g_{i}=\operatorname{det} G_{i} .
$$

We replace (4.1), (4.2) and (4.5) by (4.11), (4.13) and (4.14), then from (4.6) we obtain

$$
\int_{X_{c}} \frac{1}{A_{i}} \sum_{B_{p-1}} \sum_{\beta, r=1}^{n} \Gamma_{i, \beta \bar{\tau}} \varphi_{i}^{\beta} \bar{B}_{p-1} \cdot \varphi_{i}^{\tau \bar{B}_{p-1}} d V \leqq\|\bar{\partial} \varphi\|^{2}+\left\|\bar{\partial}^{*} \varphi\right\|^{2}
$$

for any $\varphi \in C_{0}^{0, p}\left(X_{c}, B \otimes K_{X}\right)$ with $p \geqq 1$.

We rewrite the left hand side as

$$
\int_{X_{c}} \frac{1}{A_{i}} \sum_{B_{p-1}} \sum_{\alpha, \gamma=1}^{n}\left(\sum_{\beta=1}^{n} g_{i}^{\bar{\alpha} \beta} \cdot \Gamma_{i, \beta \bar{\tau}}\right) \varphi_{i, \bar{\kappa} \bar{\beta}_{p-1}} \cdot \varphi_{i}^{\Upsilon B_{p-1}} d V .
$$

We can choose a matrix $T_{i}$ which depends, together with $T_{i}^{-1}$, differentiably on $x \in U_{i}$, satisfying $G_{i, 0}={ }^{t} T_{i} \cdot \bar{T}_{i}$. Since $G_{i}=G_{i, 0}+\Gamma_{i}$, we have $G_{i}={ }^{t} T_{i}\left\{E+{ }^{t} T_{i}^{-1} \cdot \Gamma_{i} \cdot \bar{T}_{i}^{-1}\right\} \bar{T}_{i}$. The eigenvalues of the hermitian matrix ${ }^{t} T_{i}^{-1} \cdot \Gamma_{i} \cdot \bar{T}_{i}^{-1}\left(\operatorname{resp}{ }^{t} T_{i}^{-1} \cdot \Gamma_{i, 0} \cdot \bar{T}_{i}^{-1}\right)$ are continuous functions on $X_{c}$ (resp. $X)$. From (4.12), we have

$$
{ }^{t} T_{i}^{-1} \cdot \Gamma_{i} \cdot \bar{T}_{i}^{-1} \geqq T_{i}^{-1} \cdot \Gamma_{i, 0} \cdot \bar{T}_{i}^{-1} \quad \text { on } X_{c} \cap U_{i} \text {. }
$$

Let $K^{\prime \prime}$ be a compact subset of $X_{c}$ with $K^{\prime} \subset \operatorname{Int} K^{\prime} \subset K^{\prime} \subsetneq X_{c}$. Since the closure of $X_{c}$ is compact, (4.17) implies that the first $n-q+1$ eigenvalues of the matrix ${ }^{t} T_{i}^{-1} \cdot \Gamma_{i} \cdot \bar{T}_{i}^{-1}$ taken in the order of decreasing magnitude, are positive and stay away from zero on $X_{c} \backslash K^{\prime}$. Let $x_{0} \in X_{c} \backslash K^{\prime}$ and choose a system of local coordinates $\left(z_{i}^{1}, \cdots, z_{i}^{n}\right)$ around $x_{0}$ as follows:

$$
G_{i, 0}\left(x_{0}\right)=\left(\delta_{\alpha \beta}\right) \text { and } \Gamma_{i}\left(x_{0}\right)=\left(v_{\alpha} \cdot \delta_{\alpha \beta}\right)
$$

where $\left\{v_{\alpha}\right\}_{1 \leqq \alpha \leqq n}$ are eigenvalues of the matrix ${ }^{t} T_{i}^{-1} \cdot \Gamma_{i} \cdot \bar{T}_{i}^{-1}$ at $x_{0}$ and 
satisfy $v_{1} \geqq v_{2} \geqq \cdots \geqq v_{n-q+1}>0$ and $v_{n-q+2} \geqq \cdots \geqq v_{n} \geqq 0$. Then there exists a positive constant $\varepsilon$, independent of the choice of $x_{0} \in X_{c} \backslash K^{\prime}$, such that $v_{n-q+1} \geqq \varepsilon>0$. Therefore we have

$$
G_{i}\left(x_{0}\right)^{-1} \cdot \Gamma_{i}\left(x_{0}\right)=\left(\begin{array}{ccc}
\frac{v_{1}}{1+v_{1}}, & \\
& \ddots & \\
& , \frac{v_{n}}{1+v_{n}}
\end{array}\right) \geqq \varepsilon^{\prime}\left(\begin{array}{cc}
E_{n-q+1} & 0 \\
0 & 0
\end{array}\right)
$$

where $\varepsilon^{\prime}=\frac{\varepsilon}{1+\varepsilon}$ and $E_{n-q+1}$ is the $(n-q+1, n-q+1)$ unit matrix.

We apply (4.19) to (4.16), then at $x_{0}$

$$
\begin{array}{r}
\sum_{B_{p-1}} \sum_{\alpha, \gamma=1}^{n}\left(\sum_{\beta=1}^{n} g_{i}^{\bar{\alpha} \beta} \cdot \Gamma_{i, \bar{\beta} \gamma}\right) \varphi_{i, \bar{\alpha} \bar{B}_{p-1}} \cdot \overline{\varphi_{i}^{r B_{p-1}}} \\
\geqq \varepsilon^{\prime} \sum_{B_{p-1}} \sum_{\beta=1}^{n-q+1} \varphi_{i, \bar{\beta} \bar{B}_{p-1}} \cdot \overline{\varphi_{i}^{\beta} \overline{B_{p-1}}}
\end{array}
$$

If $p \geqq q$, then $p+n-q+1 \geqq n+1$, thus any block $B_{p}$ of $p$ indices taken from $\{1,2, \cdots, n\}$ must contain one of the indices $\{1,2, \cdots, n-q+1\}$, i.e. one of the indices corresponding to the positive eigenvalues $v_{1}, v_{2}, \cdots$, $v_{n-q+1}$. It follows that

$$
\sum_{B_{p-1}} \sum_{\beta=1}^{n-q+1} \varphi_{i, \bar{\beta} \bar{B}_{p-1}} \cdot \overline{\varphi_{i}^{\Gamma B_{p-1}}} \geqq_{\beta_{1}<, \cdots,<\beta_{p}} \varphi_{i, \bar{B}_{p}} \cdot \varphi_{i}^{\bar{B}_{p}}
$$

Since the matrix $G_{i}^{-1} \cdot \Gamma_{i}$ is positive semi-definite on $X_{c}$, from (4.15), (4.16), (4.20) and (4.21) we have

$$
\|\varphi\|_{X_{c} \backslash K^{\prime}}^{2} \leqq C_{1}\left\{\|\bar{\partial} \varphi\|^{2}+\left\|\bar{\partial}^{*} \varphi\right\|^{2}\right\} \quad\left(C_{1}=\frac{1+\varepsilon}{\varepsilon}\right)
$$

for any $\varphi \in C_{0}^{0, p}\left(X, B \otimes K_{X}\right)$ with $p \geqq q$.

\section{$\S 5$. Proof of the Main Theorem}

Step 1. Vanishing Theorems on Each Sublevel Set $\boldsymbol{X}_{\boldsymbol{c}}$. Let the situations be as above. By Remark in Section 4, our base metric $d s^{2}$ is complete. Hence, by the same argument as in the proof of Theorem 3.1 and (4.22), we have

$$
\|\varphi\|_{X_{c} \backslash K^{\prime}}^{2} \leqq C_{1}\left\{\|\bar{\partial} \varphi\|^{2}+\left\|\bar{\partial}^{*} \varphi\right\|^{2}\right\}
$$


for any $\varphi \in D_{\bar{\partial}}^{0, p} \cap D_{\bar{\partial}^{\prime} p}^{0, p}$ with $p \geqq q$.

Any connected compact complex manifold $X$ is weakly 1-complete with respect to the real constant functions. Then we have $X_{c}=X$. If $X$ is non-compact, $X_{c}=\{x \in X \mid \Phi(x)<c\}$ has countable connected components. If one of them is contained in the compact subset $K^{\prime}$, it must be a compact connected component (or manifold) of $X$. Since $X$ is connected, this is a contradiction. Therefore, in our situation, the conditions of Theorem 3.1 are satisfied. Hence there exists a constant $C_{2}>0$ such that

$$
\|\varphi\|^{2} \leqq C_{2}\left\{\|\bar{\partial} \varphi\|^{2}+\left\|\bar{\partial}^{*} \varphi\right\|^{2}\right\}
$$

for any $\varphi \in D_{\vec{\partial}}^{0, p} \cap D_{\vec{\partial}^{*}, p}^{0,}$ with $p \geqq q$.

Take any $\varphi \in C^{0, p}\left(X_{c}, B \otimes K_{X}\right)$ with $\bar{\partial} \varphi=0$. We can choose a $C^{\infty}$. function $\lambda$ with the condition (4.9) such that

i) the Kähler metric $d s^{2}$ induced by (4.13) is complete,

ii) $(\varphi, \varphi)<+\infty$ (cf. [9], $\S 2$, Proof of Theorem 1).

Hence, by Corollary 3.1, we have $\varphi=\bar{\partial} \psi$ for some $\psi \in C^{0 . p-1}\left(X_{c}, B \otimes K_{Y}\right)$. Therefore we have proved that for any $c>c_{*}=\sup _{x \in K} \Phi(x)$,

$$
H^{p}\left(X_{c}, \mathcal{O}\left(B \otimes K_{X}\right)\right)=0 \quad \text { for any } p \geqq q
$$

Step 2. Approximation Lemmas. We fix two constants $d$ and $e$ such that
i) $d>e>c_{*}$,
ii) the boundary $\partial X_{e}$ of $\{x \in X \mid \Phi(x) \leqq e\}$ is smooth.

We take a $C^{\infty}$-increasing convex function $\tau(t)$ such that
i) $\tau(l):(-\infty, \infty) \rightarrow(-\infty, \infty)$,
ii) $\tau(t)=\left\{\begin{array}{ccc}0 & \text { if } & \iota \leqq \frac{1}{d-e} \\ >0 & \text { if } & t>c \\ d-e\end{array}\right.$,
iii) $\int_{0}^{+\infty} \sqrt{\tau^{\prime \prime}(t)} d t=+\infty$.

We set 


$$
\Psi=\tau\left(\frac{1}{d-\Phi}\right)
$$

We define the metrics of $B$ on $X_{d}$ by

(5.6) i) $a_{i}=a_{i, 0} \cdot \exp (\Psi)$,

ii) $\quad a_{m, i}=a_{i} \cdot \exp (m \Psi) \quad$ for any $m \geqq 0$.

We set
i) $\Gamma_{i}=\left(\Gamma_{i, \alpha \bar{\beta}}\right)$ where $\Gamma_{i, \alpha \bar{\beta}}=\frac{\partial^{2} \log a_{i}}{\partial z_{i}^{\alpha} \partial z_{i}^{\bar{\beta}}}$,
ii) $\quad \Gamma_{m, i}=\left(\Gamma_{m, i, \alpha \bar{\beta}}\right)$ where $\quad \Gamma_{m, i, \alpha \bar{\beta}}=\frac{\partial^{2} \log a_{m, i}}{\partial z_{i}^{\alpha} \partial z_{i}^{\bar{\beta}}}$ for any $m \geqq 0$.

We define a Kähler metric $d s^{2}$ on $X_{d}$ by

$$
d s^{2}=\sum_{\alpha, \beta=1}^{n}\left(g_{i, \alpha \bar{\beta}, 0}+\Gamma_{i, \alpha \bar{\beta}}\right) d z_{i}^{\alpha} \cdot d z_{i}^{\bar{\beta}}
$$

Remark. By the choice (5.5), $d s^{2}$ is a complete Kähler metric as in Remark in Section 4.

We set

$$
G_{i}=\left(g_{i, \alpha \bar{\beta}}\right) \quad \text { where } \quad g_{i, \alpha \bar{\beta}}=g_{i, \alpha \bar{\beta}, 0}+\Gamma_{i, \alpha \bar{\beta}} .
$$

Using (5.6), we define the metrics of $B \otimes K_{X}$ on $X_{d}$ :

i) $A_{i}=a_{i} \cdot g_{i}$,

ii) $A_{m, i}=a_{m, i} \cdot g_{i}$ for any $m \geqq 0$, where $g_{i}=\operatorname{det} G_{i}$.

For any integer $m \geqq 0$, we define

$$
\begin{aligned}
& (\varphi, \psi)_{m}=(\varphi, \psi)_{m \Psi} \\
& \|\varphi\|_{m}^{2}=(\varphi, \varphi)_{m}
\end{aligned}
$$

for any $\varphi, \psi \in L^{0, p}\left(X_{d}, B \otimes K_{X}, m \Psi\right)$. We denote the formal adjoint of $\bar{\partial}$ with respect to the inner product $(\varphi, \psi)_{m}$ by $\vartheta_{m}$ and the adjoint operator in $L^{0 \cdot} \cdot\left(X_{d}, B \otimes K_{X}, m \Psi\right)$ by $\bar{\partial}_{m}^{*}$.

Now we have 


$$
G_{i}^{-1} \cdot \Gamma_{i} \leqq G_{i}^{-1} \cdot \Gamma_{m, i} \quad \text { for any } m \geqq 0 \text {. }
$$

Hence by the same argument as in Section 4 , we have, for any $m \geqq 0$,

$$
\|\varphi\|_{m X_{d} \backslash K^{\prime}}^{2} \leqq C_{1}\left\{\|\bar{\partial} \varphi\|_{m}^{2}+\left\|\bar{\partial}_{m}^{*} \varphi\right\|_{m}^{2}\right\}
$$

for any $\varphi \in D_{\vec{\partial}}^{0, p} \cap D_{\bar{\partial}^{\prime} m}^{n, p}$ with $p \geqq q$, where $C_{1}>0$ is independent of $m$ and $K^{\prime}$ is a compact subset with $K \subset \operatorname{Int} K^{\prime} \subset K^{\prime} \subsetneq X_{d}$. Then, for each $m$, we have a positive constant such that (5.2) holds. In general, this constant depends on $m$. The basic idea of the following lemma is due to Hörmander [5]. (Compare with [10], Proposition 4.2.)

Lemma 5. 1. There exists $m_{0}$ and $C_{0}>0$ such that for any $m \geqq m_{0}$ and $p \geqq q$,

$$
\|\varphi\|_{m}^{2} \leqq C_{0}\left\{\|\bar{\partial} \varphi\|_{m}^{2}+\left\|\bar{\partial}_{m}^{*} \varphi\right\|_{m}^{2}\right\},
$$

provided $\varphi \in D_{\overline{\bar{o}}}^{0 . p} \cap D_{\bar{\partial} \bar{m}}^{0, p} \subset L^{0, p}\left(X_{d}, B \otimes K_{X}, m \Psi\right)$.

Proof. Assume that the assertion is false. There would be a sequence $\left\{\varphi_{k}\right\}$ such that

$$
\begin{aligned}
& \text { i) } \varphi_{k} \in D_{\overline{\bar{\partial}}}^{0, p} \cap D_{\bar{\partial} \bar{k}}^{0, p} \subset L^{0, p}\left(X_{d}, B \otimes K_{X}, k \Psi\right), \\
& \text { ii) }\left\|\varphi_{k}\right\|_{k}^{2}=1, \\
& \text { iii) }\left\|\bar{\partial} \varphi_{k}\right\|_{k}^{2},\left\|\bar{\partial}_{k}^{*} \varphi_{k}\right\|_{k}^{2} \rightarrow 0 \quad \text { as } \quad k \rightarrow+\infty .
\end{aligned}
$$

Let $g_{k}=e^{-k y} \cdot \varphi_{k}$, then we have

$$
\begin{aligned}
& \text { i) } \bar{\partial}^{*} g_{k}=e^{-k \psi^{*}} \bar{\partial}_{k}^{*} \varphi_{k}, \\
& \text { ii) }\left\|\bar{\partial}^{*} g_{k}\right\|_{-k}=\left\|\bar{\partial}_{k}^{*} \varphi_{k}\right\|_{k} .
\end{aligned}
$$

By (5.11), we have

$$
\left\|g_{k}\right\| \leqq\left\|g_{k}\right\|_{-k}=\left\|\varphi_{k}\right\|_{k}=1
$$

Therefore choosing a subsequence if necessary, we may assume that $\left\{g_{k}\right\}$ has a weak limit $g$ in $L^{0, p}\left(X, B \otimes K_{X}\right)$. On the other hand, it follows that

$$
\left\|y_{k}\right\|_{X_{d} \backslash K^{\prime}}^{2} \leqq\left\|\varphi_{k}\right\|_{k X_{d} \backslash X^{\prime}}^{2} \leqq C_{1}\left\{\left\|\bar{\partial} \varphi_{k}\right\|_{k}^{2}+\left\|\bar{\partial}_{k}^{*} \varphi_{k}\right\|_{k}^{2}\right\} .
$$

By (5.11) 


$$
\lim _{k \rightarrow+\infty}\left\|g_{k}\right\|_{X_{d} \backslash K^{\prime}}^{2}=0
$$

Hence we have $\left.g\right|_{X_{d} \backslash K^{\prime}} \equiv 0$. Then it follows that

$$
\text { supp } g \subseteq K^{\prime} .
$$

From (5.11), (5.12) and (5.13), we have $\bar{\partial} g=0$ and $\bar{\partial}^{*} g=0$ in $L^{0, p+1}\left(X_{e}, B \otimes K_{X}\right)$ and $L^{0, p-1}\left(X_{e}, B \otimes K_{X}\right)$ respectively. Since any connected component of $X_{c}$ is not contained in $K^{\prime}$, by Theorem 3. 2, we have

$$
g=0 .
$$

By (5.11), we may assume that $\left\{g_{k}\right\}$ is strongly convergent on $K^{\prime}$. (5.14) implies that the limit is zero on $K^{\prime}$. From (5.10) and (5.11), we obtain a contradiction.

q.e.d.

Lemma 5. 2. If $\phi \in L^{0, p}\left(X_{e}, B \otimes K_{X}\right)$ with $p \geqq q-1$ and $\bar{\partial} \psi=0$, then for any $\varepsilon>0$, there exists $\tilde{\psi} \in L^{0, p}\left(X_{d}, B \otimes K_{X}\right)$ such that $\left\|\left.\widetilde{\psi}\right|_{X_{c}}-\psi\right\|_{X_{e}}^{2}<\varepsilon$ and $\bar{\partial} \widetilde{\psi}=0$.

Proof. It suffices to show that if $u \in L^{0, p}\left(X_{e}, B \otimes K_{X}\right)$ and

$$
\int_{X_{e}}\langle f, u\rangle d V=0
$$

for any $f \in L^{0, p}\left(X_{d}, B \otimes K_{x}\right)$ with $\vec{\partial} f=0$, then we have

$$
\int_{X_{e}}\langle g, u\rangle d V=0
$$

if $g \in L^{0, p}\left(X_{e}, B \otimes K_{X}\right)$ and $\bar{\partial} g=0$.

Extend the definition of $u$ by setting $u=0$ on $X_{d} \backslash X_{e}$. We denote it by $u^{\prime}$. Then (5.15) implies that $u^{\prime}$ is orthogonal to $N_{\vec{a}}^{0, p} \subset L^{0, p}\left(X_{d}, B\right.$ $\left.\otimes K_{X}, m \Psi\right)$ for any $m$, we have $u^{\prime} \in \overline{R_{\bar{\partial} m}^{0, p}} \subset L^{0, p}\left(X_{d}, B \otimes K_{X}, m \Psi\right)$. The condition $R_{\vec{\partial}_{m}^{*}}^{0, p}=\overline{R_{\bar{\partial}_{m}^{\prime}}^{0, p}}$ is equivalent to $R_{\bar{\partial}}^{0, p+1}=\overline{R_{\vec{b}}^{0, p+1}}$ (cf. [5], Theorem 1.1.1). By (5.10), we have $R_{\bar{\partial}}^{0, p+1}=\overline{R_{\bar{\partial}}^{0, p+1}} \subset L^{0, p+1}\left(X_{d}, B \otimes K_{X}, m \Psi\right)$ for $m \geqq 0$ and $p \geqq q-1$. Hence, from Lemma 5.1, for any $m \geqq m_{0}$ we have

$$
\iota^{\prime}=\bar{\partial}_{m}^{*} v_{m}
$$

for some $v_{m} \in L^{0, p-1}\left(X_{d}, B \otimes K_{X}, m \Psi\right)$ with $\left\|v_{m}\right\|_{m}^{2} \leqq C_{0} \cdot\left\|u^{\prime}\right\|^{2}$.

We set 


$$
\mathfrak{w}_{m}=e^{-m y} \cdot v_{m} \quad \text { for } m \geqq m_{0},
$$

then

$$
\left\|w_{m}\right\|^{2} \leqq\left\|w_{m}\right\|_{-m}^{2}=\left\|v_{m}\right\|_{m}^{2} \leqq C_{0} \cdot\left\|u^{\prime}\right\|^{2} .
$$

Hence $\left\{w_{m}\right\}$ has a subsequence which is weakly convergent in $L^{0, p-1}\left(X_{d}, B\right.$ $\left.\otimes K_{X}\right)$. Let the weak limit be $w$. On the other hand, for every $\varepsilon>0$

$$
\int_{\left\{r \in X_{d}^{\prime} \mid \Psi(x)>\varepsilon\right\}} e^{m \Psi}\left\langle w_{m}, w_{m}\right\rangle d V \leqq C_{0}\left\|u^{\prime}\right\|
$$

and we have

$$
e^{m \varepsilon} \int_{\left\{x \in X_{o} \mid W^{\prime}(x) \geq \varepsilon\right\}}\left\langle w_{m}, w_{m}\right\rangle d V \leqq C_{0}\left\|u^{\prime}\right\|
$$

It follows that $\int_{\left\{x \in X_{d} \mid \Psi(x) \geq \varepsilon\right\}}\left\langle w_{m}^{\prime}, w_{m}\right\rangle d V$ tends to zero, and hence $w_{m} \rightarrow 0$ almost everywhere in $\left\{x \in X_{d} \mid \Psi(x) \geqq \varepsilon\right\}$. Hence $w=0$ on $\left\{x \in X_{d} \mid \Psi(x)\right.$ $\geqq \varepsilon\}$ for every $\varepsilon>0$. Therefore we have

$$
\text { supp } w \subseteq \bar{X}_{e} \text { and } \bar{\partial}^{*} w=u^{\prime} \text {. }
$$

Since $\bar{X}_{e}$ is compact and $\partial X_{e}$ is smooth, from [5] Proposition 1. 2. 3, there exists a sequence $\left\{w^{k}\right\}$ such that $\left\{w^{k}\right\} \subset C_{0}^{0, p+1}\left(X_{e}, B \otimes K_{X}\right)$ and $\left\|w^{k}-w\right\|_{X_{e}}^{2}$, $\left\|\bar{\partial}^{*} w^{k}-\bar{\partial}^{*} w\right\|_{X_{e} \rightarrow 0}^{2}$ as $k \rightarrow+\infty$.

We have, for any $v \in D_{\vec{\partial}}^{0, p} \subset L^{0, p}\left(X_{e}, B \otimes K_{X}\right)$,

$$
\begin{aligned}
\left(\bar{\partial} v,\left.w\right|_{X_{e}}\right)_{x_{e}} & =\lim _{k \rightarrow+\infty}\left(\bar{\partial} v, w^{k}\right)_{x_{e}}=\lim _{k \rightarrow+\infty}\left(v, \bar{\partial}^{*} w^{k}\right)_{x_{e}} \\
& =\left(v, \bar{\partial}^{*}\left(\left.w\right|_{x_{e}}\right)\right)_{x_{\epsilon}} .
\end{aligned}
$$

Hence

$$
\bar{\partial}^{*}\left(\left.w\right|_{x_{e}}\right)=u
$$

Therefore, if $g \in L^{0, p}\left(X_{e}, B \otimes K_{X}\right)$ and $\bar{\partial} g=0$, we have

$$
\int_{x_{e}}\langle g, u\rangle d V=\int_{x_{e}}\langle\bar{\partial} g, w\rangle d V=0 . \quad \text { u.c.d. }
$$

If in particular $q=1$, replacing $L^{0, p}\left(X_{d}, B \otimes K_{X}\right)\left(\right.$ resp. $L^{0, p}\left(X_{c}, B \otimes K_{X}\right)$ ) by $\Gamma\left(X_{d}, \mathcal{O}\left(B \otimes K_{X}\right)\right)\left(\right.$ resp. $\left.\Gamma\left(\bar{X}_{e}, \mathcal{O}\left(B \otimes K_{X}\right)\right)\right)$, we can prove the following in the same way as we proved Lemma 5.2.

Lemma 5. 3. Let $X_{d}$ and $X_{e}$ be as above and let a holomorphic 
line bundle $B$ be positive on $X \backslash K$ and semi-positive on $X$. Then for any holomorphic section $\varphi \in \Gamma\left(\bar{X}_{e}, \mathcal{O}\left(B \otimes K_{X}\right)\right), \bar{X}_{e}$ being the closure of $X_{e}$ in $X$, and for any $\varepsilon>0$, there exists a section $\tilde{\varphi} \in \Gamma\left(X_{d}, \Theta\left(B \otimes K_{X}\right)\right)$ such that $\|\tilde{\varphi}-\varphi\|_{X_{c}}^{2}<\varepsilon$.

Let $C$ be a compact subset of $X_{d}$. We set $|\varphi|_{C}=\sup _{x \in C} \sqrt{\langle\varphi, \varphi\rangle(x)}$ for $\varphi \in \Gamma\left(X_{d}, \mathcal{O}\left(B \otimes K_{X}\right)\right)$, where $\langle\varphi, \varphi\rangle=A_{i}^{-1}\left|\varphi_{i}\right|^{2}$ (see (5.8)). Then, using Cauchy's integral formula in each local coordinate $U_{i}$ with $U_{i} \cap C$ $\neq \emptyset$, we can find a positive constant $M$ such that

$$
|\varphi|_{C} \leqq M\|\varphi\|_{C}
$$

Hence we obtain the following.

Lemma 5. 4. Let $X_{d}$ and $X_{e}$ be as above. Let a holomorphic line bundle $B$ be positive on $X \backslash K$ and semi-positive on $X$. Then for any holomorphic section $\varphi \in \Gamma\left(\bar{X}_{e}, \mathcal{O}\left(B \otimes K_{X}\right)\right)$ and for any $\varepsilon>0$, there exists a section $\tilde{\varphi} \in \Gamma\left(X_{d}, \mathcal{O}\left(B \otimes K_{X}\right)\right)$ such that $|\widetilde{\varphi}-\varphi|_{X_{e}}<\varepsilon$.

Step 3. Global Vanishing Theorems. By Sard's theorem, we can choose a sequence $\left\{c_{\nu}\right\}_{\nu=0,1, \ldots}$, of real numbers such that

i) $c_{0}>c_{*}$,

ii) $c_{\nu \perp 1}>c_{\nu}$ and $c_{\nu} \rightarrow \infty$ as $\nu \rightarrow+\infty$,

iii) the boundary $\partial X_{c_{\nu}}$ of $\left\{x \in X \mid \Phi(x) \leqq c_{\nu}\right\}$ is smooth for any $\nu \geqq 0$.

For any pair $\left(c_{\nu+2}, c_{\nu}\right)(\nu \geqq 0)$, we choose a $C^{\infty}$ increasing convex function $\tau_{\nu+2}$ such that
i) $\tau_{\nu+2}(t):(-\infty, \infty) \rightarrow(-\infty, \infty)$,
ii) $\tau_{\nu+2}(t)=\left\{\begin{array}{rll}0 & \text { if } & t \leqq \frac{1}{c_{\nu+2}-c_{\nu}} \\ >0 & \text { if } & t>\frac{1}{c_{\nu+2}-c_{\nu}},\end{array}\right.$
iii) $\int_{0}^{+\infty} \sqrt{\tau_{\nu+2}^{\prime \prime}(t)} d t=+\infty$. 
We set

$$
\begin{aligned}
& X_{\nu}=\left\{x \in X \mid \Phi(x)<c_{\nu}\right\}, \\
& \Psi_{\nu+2}=\tau_{\nu+2}\left(\frac{1}{c_{\nu+2}-\Phi}\right)
\end{aligned}
$$

for any $\nu \geqq 0$. Then, for any pair $\left(c_{y+2}, c_{\nu}\right)$, Lemma 5.2 and Lemma 5.4 hold.

The case $q=1$. $\mathscr{X}=\left\{X_{\nu}\right\}_{\nu \geq 0}$ is a covering of $X$. For any $\nu \geqq 1$, we set $\mathscr{X}_{\nu}=\left\{X_{\mu}\right\}_{\mu \leqq \nu}$, then $\mathscr{X}_{\nu}$ is a covering of $X_{\nu}$. By (5.3), $\mathscr{X}$ (resp. $\mathfrak{X}_{\nu}$ ) is a Leray covering for the sheaf $\mathcal{O}\left(B \otimes K_{X}\right)$ on $X$ (resp. $X_{\nu}$ ). Then we have, for any $i \geqq 1$ and $\nu \geqq 1$,

$$
H^{i}\left(X, \circlearrowleft\left(B \otimes K_{X}\right)\right)=H^{i}\left(\mathscr{X}, \mathcal{O}\left(B \otimes K_{X}\right)\right)
$$

and

$$
H^{i}\left(\mathfrak{X}_{\nu}, \mathcal{O}\left(B \otimes K_{X}\right)\right)=H^{i}\left(X_{\nu}, \mathcal{O}\left(B \otimes K_{X}\right)\right)=0 .
$$

Let $\sigma \in Z^{i}\left(\mathscr{X}, \odot\left(B \otimes K_{X}\right)\right), i \geqq 1$. Let $\sigma_{\nu}$ be the restriction of $\sigma$ to $X_{\nu}$. Then $\sigma_{\nu} \in Z^{i}\left(\mathscr{X}_{\nu}, \mathcal{O}\left(B \otimes K_{X}\right)\right)$ so there is an $\alpha_{\nu} \in C^{i-1}\left(\mathscr{X}_{\nu}, \ominus\left(B \otimes K_{X}\right)\right)$ such that $\delta \alpha_{\nu}=\sigma_{\nu}$. As an element of $C^{i-1}\left(\mathscr{X}_{\nu-1}, \mathcal{O}\left(B \otimes K_{X}\right)\right), \delta \alpha_{\nu}=\delta \alpha_{\nu-1}$, and thus $\alpha_{\nu}-\alpha_{\nu-1}^{\prime} \in Z^{i-1}\left(\mathfrak{X}_{\nu-1}, \mathcal{O}\left(B \otimes K_{X}\right)\right)$.

When $i>1$. Since $\alpha_{\nu}-\alpha_{\nu-1} \in Z^{i-1}\left(X_{\nu-1}, O\left(B \otimes K_{x}\right)\right)$, there is a $\beta_{\nu-1}$ $\in C^{i-2}\left(\mathscr{X}_{\nu-1}, \mathcal{O}\left(B \otimes K_{X}\right)\right)$ such that $\delta \beta_{\nu-1}=\alpha_{\nu}-\alpha_{\nu-1}$ on $X_{\nu-1}$. Define $\alpha \in C^{i-1}\left(\mathfrak{X}, \mathcal{O}\left(B \otimes K_{X}\right)\right)$ as follows:

$$
\alpha=\alpha_{\nu}-\delta\left(\sum_{n<\nu} \beta_{\mu}\right) \quad \text { on } Y_{\nu} .
$$

It is easily verified that $\alpha$ is well defined. Finally, for any $\nu, \delta \alpha=$ $\delta \alpha_{\nu}-\delta \delta\left(\sum_{\mu<\nu} \beta_{\mu}\right)=\delta \alpha_{\nu}=\sigma_{\nu}$. Hence we have $\delta \alpha=\sigma$.

When $i=1$. Since $\alpha_{\nu}-\alpha_{\nu-1} \in \Gamma\left(X_{\nu-1}, \mathcal{O}\left(B \otimes K_{X}\right)\right)$, by L.emma 5.4 we can find, for any $\varepsilon>0$, a $r \in \Gamma\left(X_{\nu}, \mathcal{O}\left(B \otimes K_{X}\right)\right)$ such that $\mid \alpha_{\nu}-\alpha_{\nu-1}$ $-\left.\gamma\right|_{\bar{X}_{y-2}}<\varepsilon$. Therefore, inductively, we have a sequence $\left\{\lambda_{\nu}\right\}_{\nu \geqq 1}$ so that

$$
\begin{aligned}
& \text { i) } \lambda_{\nu} \in C^{0}\left(\mathscr{X}_{\nu}, \Theta\left(B \otimes K_{X}\right)\right) \text { and } \lambda_{1}=\alpha_{1}, \\
& \text { ii) } \delta \lambda_{\nu}=\sigma_{\nu}, \\
& \text { iii) }\left|\lambda_{\nu+1}-\lambda_{\nu}\right|_{\bar{x}_{\nu-1}}<2^{-\nu} .
\end{aligned}
$$


For any $\nu, \lim _{\mu \geq \nu} \lambda_{\mu}$ defines an element of $C^{0}\left(\mathscr{X}_{\nu}, \mathcal{O}\left(B \otimes K_{X}\right)\right)$ and clearly this limit is the same as the restriction of $\lim _{\mu \geqq \eta} \lambda_{\mu}$ for any $\eta \geqq \nu+1$. Thus we can define an element $\lambda$ of $C^{0}\left(\mathscr{X}, \Theta\left(B \otimes K_{X}^{\mu \geq \eta}\right)\right)$ by $\lambda=\lim _{\nu \rightarrow+\infty} \lambda_{\nu}$. For any $\nu, \delta\left(\lim _{\mu \geq \nu} \lambda_{\mu}\right)=\lim _{\mu \geqq \nu} \delta \lambda_{\mu}=\sigma_{\nu}$. Hence we have $\delta \lambda=\sigma$.

The case $q>1$. We denote by $L_{1 \mathrm{loc}}^{0, p}\left(X, B \otimes K_{X}\right)$ the set of the locally square integrable $(0, p)$ forms on $X$ with values in $B \otimes K_{X}$. For $p \geqq 1$, there is a natural isomorphism

(5. 23) $\quad H^{p}\left(X, \odot\left(B \otimes K_{Y}\right)\right)$

$$
\cong \frac{\left\{f \in L_{\mathrm{loc}}^{0, p}\left(X, B \otimes K_{X}\right) ; \bar{\partial} f=0\right\}}{\left\{f \in L_{\mathrm{loc}}^{0, p}\left(X, B \otimes K_{X}\right) ; f=\bar{\partial} g \text { for some } g \in L_{\mathrm{loc}}^{0, p-1}\left(X, B \otimes K_{X}\right)\right\}} .
$$

Therefore, for $p \geqq q$, it suffices to show that for any $\varphi \in L_{\text {loc }}^{0, p}\left(X, B \otimes K_{Y}\right)$ with $\bar{\partial} \varphi=0$, there exists a $\psi \in L_{\mathrm{loc}}^{0, p-1}\left(X, B \otimes K_{X}\right)$ such that $\bar{\partial} \psi=\varphi$.

In this proof, for any $\nu \geqq 0$, we set

$$
\begin{aligned}
& \text { i) } \varphi_{\nu}=\left.\varphi\right|_{X_{\nu}}, \\
& \text { ii) } L^{0, p}\left(X_{\nu+2}, B \otimes K_{X}, \Psi_{\nu+2}\right)=L^{0, p}\left(X_{\nu+2}, B \otimes K_{X}\right), \\
& \text { iii) } \quad L^{0, p}\left(X_{\nu}, B \otimes K_{X}, 0\right)=L^{0, p}\left(X_{\nu}, B \otimes K_{X}, \Psi_{\nu+2}\right), \\
& \text { iv) }\|f\|_{\nu+2}^{2}=\int_{X_{\nu+2}}\langle f, f\rangle e^{-\psi_{\nu+2}} d V \\
& \text { for } f \in L^{0, p}\left(X_{\nu+2}, B \otimes K_{X}, \Psi_{\nu+2}\right)
\end{aligned}
$$

where $\langle f, f\rangle=\left(a_{i, 0} \cdot g_{i}\right)^{-1} \sum_{B_{p}} f_{i, \bar{B}_{p}} \cdot f_{i}^{\bar{B}_{p}}$.

Then $\varphi_{\nu} \in L^{0, p}\left(X_{\nu}, B \otimes K_{X}, \Psi_{\nu}\right)$ and $\bar{\partial} \varphi_{\nu}=0(\nu \geqq 2)$. Hence there exists a $\psi_{\nu}^{\prime} \in L^{0, p-1}\left(X_{\nu}, B \otimes K_{X}, \Psi_{\nu}\right)$ such that $\bar{\partial} \psi_{\nu}^{\prime}=\varphi_{\nu}$ for any $\nu \geqq 2$. We now choose, by induction, a sequence $\left\{\psi_{\nu}\right\}_{\nu \geq 1}$ so that

$$
\begin{aligned}
\text { i) } & \psi_{\nu} \in L_{\mathrm{loc}}^{0, p}\left(X, B \otimes K_{X}\right) \\
\text { ii) } & \bar{\partial} \psi_{\nu}=\varphi_{\nu} \text { on } X_{\nu} \\
\text { iii) } & \left\|\psi_{\nu+1}-\psi_{\nu}\right\|_{\nu+2, X_{\nu}}^{2}<2^{-\nu} .
\end{aligned}
$$

We set

$$
\phi_{1}=\left\{\begin{array}{cl}
\left.\phi_{2}^{\prime}\right|_{X_{1}} & \text { on } X_{1}^{r} \\
0 & \text { on } X \backslash X_{1} .
\end{array}\right.
$$


Since $\quad \psi_{2}^{\prime} \in D_{\vec{\partial}}^{0, p-1} \subset L^{0, p-1}\left(X_{2}, B \otimes K_{X}, \Psi_{2}\right)$, we have $\psi_{1} \in D_{\vec{\partial}}^{0, p-1} \subset L^{0, p-1}$ $\left(X_{1}, B \otimes K_{X}, 0\right)$ and $\bar{\partial} \psi_{1}=\varphi_{1}$. Suppose $\psi_{1}, \cdots, \psi_{\nu-1}$ are chosen. Then

$$
\left.\left(\psi_{\nu+1}^{\prime}-\psi_{\nu-1}\right)\right|_{x_{\nu-1}} \in L^{0, p-1}\left(X_{\nu-1}, B \otimes K_{X}, 0\right)
$$

and

$$
\left.\bar{\partial}\left(\psi_{\nu+1}^{\prime}-\psi_{\nu-1}\right)\right|_{X_{\nu-1}}=0
$$

By Lemma 5. 2, there exists a $g \in L^{0, p-1}\left(X_{y+1}, B \otimes K_{X}, \Psi_{\nu+1}\right)$ such that $\left\|g-\left(\psi_{\nu+1}^{\prime}-\psi_{\nu-1}\right)\right\|_{\nu+1}^{2}, x_{\nu-1}<2^{-(\nu 1)}$ and $\bar{\partial} g=0$.

We set

$$
\psi_{\nu}=\left\{\begin{array}{cl}
\left.\phi_{\nu+1}^{\prime}\right|_{x_{\nu}}-\left.g\right|_{X_{\nu}} & \text { on } X_{\nu} \\
0 & \text { on } X \backslash X_{\nu}
\end{array}\right.
$$

Then we have

$$
\begin{aligned}
& \text { i) } \phi_{\nu} \in D_{\bar{\partial}}^{0, p-1} \subset L^{0, p-1}\left(X_{\nu}, B \otimes K_{X}, 0\right) \\
& \text { ii) } \bar{\partial} \psi_{\nu}=\varphi_{\nu} \\
& \text { iii) }\left\|\psi_{\nu}-\psi_{\nu-1}\right\|_{\nu+1, X_{\nu-1}}^{2}<2^{-(\nu-1)} .
\end{aligned}
$$

From (5.26), for any $\nu,\left\{\psi_{\mu}\right\}_{\mu \geqq \nu}$ converges with respect to the norm \|\|$_{\nu}$, and clearly the limit is the same as the restriction of $\lim _{\mu \geq \eta} \psi_{\mu}$ for any $\eta \geqq \nu+1$. Thus we can define an element $\psi$ of $L_{\mathrm{loc}}^{0, p-1}\left(X, B \otimes K_{Y}\right)$ by $\psi=\lim _{\nu \rightarrow+\infty} \psi_{\nu}$.

$$
\text { For every } \nu \geqq 1 \text {, }
$$

$$
\begin{aligned}
& \text { i) } \lim _{\mu \geqq \nu} \psi_{\mu}=\psi \text { in } L^{0, p-1}\left(X_{\nu}, B \otimes K_{X}, 0\right), \\
& \text { ii) }\left.\lim _{\mu \geqq \nu} \bar{\partial} \psi_{\mu}\right|_{X_{\nu}}=\varphi_{\nu} \quad \text { in } L^{0, p}\left(X_{\nu}, B \otimes K_{X}, 0\right) .
\end{aligned}
$$

Since $\bar{\partial}$ is a closed operator in $L^{0, p-1}\left(X_{\nu}, B \otimes K_{X}, 0\right)$ for every $\nu \geqq 1$, we have, for any $\nu \geqq 1$,

$$
\bar{\partial} \psi=\varphi_{\nu} \quad \text { in } L^{n, p}\left(X_{y}, B \otimes K_{.}, 0\right) .
$$

Hence we have

$$
\bar{\partial} \psi=\varphi \quad \text { in } L_{\mathrm{loc}}^{0, p}\left(X, B \otimes K_{X}\right)
$$

q.e.d. 


\section{References}

[1] Abdelkader, O., Vanishing of the cohomology of a weakly 1-complete Kähler manifold with value in a semi-positive vector bundle, C. R. Acad. Sci. Paris, 290 (1980), 75-78.

[2] Aronszajn, N., A unique continuation theorem for solutions of elliptic partial differential equations or inequalities of second order, J. Math. Pur. Appl., 36 (1957), 235-249.

[3] Folland, G. B. and Kohn, J. J., The Neumann problem for the Cauchy-Riemann complex, Ann. Math. Studies, 75, 1972.

[4] Grauert, H. and Riemenschneider, O., Kähler Mannigfaltigkeiten mit hyper-qkonvexem Rand, in Gunning, G. C.: Problem in analysis, paper in honor of $S$. Bochner, Princeton Univ. Press, 1970, 61-79.

[5] Hörmander, L., $L^{2}$ estimates and existence theorems for the $\bar{\partial}$ operator, Acta. Math., 113 (1965), 89-152.

[6] Hörmander, L., An introduction to complex analysis in several variables, D. Van Nostrand, Princeton, N. J., 1966.

[7] Kodaira, K., On a differential-geometric method in the theory of analytic stacks, Proc, Nat. Acad. Sci., U.S. A., 39 (1953), 1268-1273.

[8] Nakano, S., On the inverse of monoidal transformation, Publ. RIMS, Kyoto Univ., 6 (1970-71), 483-502.

[9] Nakano, S., Vanishing theorems for weakly l-complete manifolds, II, Publ. RIMS, Kyoto Univ., 10 (1974), 101-110.

[10] Ohsawa, T., Finiteness theorems on weakly l-complete manifolds, Publ. RIMS, Kyoto Univ., 15 (1979), 853-870.

[11] Riemenschneider, O., Characterizing Moisezon spaces by almost positive coherent analytic sheaves, Math. Z., 123 (1971), 263-284.

[12] Vesentini, E., Lectures on Levi convexity of complex manifolds and cohomology vanishing theorems, Tata Institute of Fundamental Research, Bombay, 1967.

Added in proof: The author and T. Ohsawa have proved that the global vanishing thheorem of Theorem 2 holds i.e. $H^{p}\left(X, \Omega^{q}(B)\right)=0$ for $p+q \geq n+k$. See "A vanishing theorem for $H^{p}\left(X, \Omega^{q}(B)\right)$ on weakly 1-complete manifolds", forthcoming. 